\begin{tabular}{c} 
International Journal of Engineering \& Technology, 7 (3) (2018) 1276-1281 \\
International Journal of Engineering \& Technology \\
SPC \\
Website: $\begin{array}{c}\text { www.sciencepubco.com/index.php/IJET } \\
\text { doi: } 10.14419 / \text { ijet.v7i3.13104 } \\
\text { Research paper }\end{array}$ \\
\hline
\end{tabular}

\title{
Control and communication of renewable energy based smart grid: an overview
}

\author{
S. Surender Reddy * \\ Department of Railroad and Electrical Engineering, Woosong University, Daejeon, Republic of Korea \\ *Corresponding author E-mail: surender@wsu.ac.kr
}

\begin{abstract}
Smart Grid (SG) involves complex controls and communications. These controls include both the local as well as global controls. SG involves a two-way communication system at each stage and that is one of the advantages over the conventional utility grid. However, due to this two-way communications, the issues such as complexity, data gathering and management, etc, are introduced into the SG system. Therefore, this new grid requires a more complex and control operation. The objective of this paper is to evaluate various control systems, communication technologies, and real time measurement tools that are involved in the SG. This paper also performs the cost benefit analysis (CBA) of the control and communication schemes involved in the SG. The paper evaluates the latest tools for control and communications that are available, and it also evaluates the issues and challenges relative to it.
\end{abstract}

Keywords: Smart Grid; Grid Control; Communication; Renewable Energy Sources; Smart Metering; Cost Benefit Analysis; Stability.

\section{Introduction}

Smart Grid (SG) is similar to a conventional utility grid in all aspects, however, new real time communication devices and advanced control devices added to the conventional grid and it becomes the Smart Grid. Renewable energy is the need for today's grid. With the growing demand every day, it is almost impossible to meet the supply and demand gap without this renewable energy. Moreover, the renewable energy is an unlimited source of energy and with almost no pollution. Power electronic devices are also plays a vital role in the SG [1]. With the varieties of energy sources, the power electronics play an important part in energy conversion. The renewable energy is produced through the harnessing of natural products. Usually, it does not create pollution. Moreover, it is available in unlimited amount. Renewable energy is key in the generation mix to meet the energy demand and the decarburization targets. Renewable energy production is increasing gradually and it is replacing conventional sources as main stream sources in many areas of world. It sometimes suffers from reliability problem but it can be solved with efficient storage options [2-3].

The main concern about the integration of renewable energy sources (RESs) to the utility grid are their variability and uncertain. Therefore, they need a prominent control technologies for the deployment of RESs, for their reliable and high performance operation [4]. Reference [5] describes the review of recent literature on the integration of RESs to the SG system. Reference [6] presents an approach for the optimal use of SG technologies to integrate RESs into the existing electrical grid, by considering their technical status, applicability, costs and market maturity for different uses. Reference [7] explores the potential applications of Power Line Communication-based Advanced Metering Infrastructure (AMI) system beyond metering of the available channel. A comprehensive review of energy management strategies and grid integration of microgrids is presented in [8]. An overview of various renewable energy sources (RESs) and their efficient utilization are presented in [9].

Reference [10] presents the security and control aspects of the SG, by including the SG architecture, communications and networking features. The SG system and its implementations using the Industrial, Scientific and Medical (ISM) band is described in [11]. Reference [12] introduces about the SG and it's associated socioeconomic, environmental, technical and other non-tangible benefits to the society. The implementation of home energy management system and it's relation with the SG technology, and it's role improve the reliability of power systems is described in [13]. The challenges of system operators and energy planners, i.e., technical, economic, policy, and regulatory are addressed in [14]. The objective of reference [15] is to disclose in a clear and clean way that what SG is and what kind of communication methods are used. The requirement of Information and Communication Technology to ensure a two-way communications to collect data from the meters and sensors located in the grid is presented in [16].

The effectiveness and implementation of a SG depends mostly on the proper development and utilization of a communication and networking system, which controls, monitors and manages the operations of the utility grid. The aim of this paper is to review the control and communication technologies of a system in the context of SGs and RESs. This paper also discusses the advanced control and communication techniques that optimize the operation of RESs based SGs.

The rest of this paper is organized as follows: Sections 2 and 3 presents the control and communication aspects of renewable energy based Smart Grid (SG), respectively. The computation and performance assessments of SGs are presented in Section 4. The cost benefit analysis of control and communication technologies is presented in Section 5. Finally, the conclusions are presented in Section 6. 


\section{Control in renewable energy based smart grid}

The control is a basic part of both conventional and smart grids. Especially, the Smart Grid (SG) involves many communication and real time devices. The SG is conceptualized as a cyberphysical system which facilitates and supports the enhanced responsiveness and controllability of highly distributed resources within the power systems. Moreover, with advent of new tools such as Phasor Measurement Units (PMUs), the control can be local as well as global. The involvement of RESs makes it more difficult due to the uncertainty involved with it. Therefore, the new control strategies with new optimization tools such as adaptive dynamic programming, stochastic programming, heuristic programming and evolutionary programming are suggested by researchers as a solution to this problem [17]. The use of RESs as distributed generation burdens the Distributed Managements System (DMS) with some activities of Energy Management System (EMS). The DMS is now more complicated in the sense that it needs to be coordinated with the EMS to economically dispatch its distributed energy resources, while maintaining the quality and reliability of power delivery.
Moreover, the SG involves local as well as global control schemes Sometimes, fault doesn't last for a long time and it does not involve large part of the grid. Hence, during such condition, the system operator (SO) that controls the grid centrally does not need to notify and he should not be held responsive for such micro level control operation. If local control settings are strong enough, such faults can be solved in minimum time with least affected area. However, some major faults that can affect the whole system and if not attended immediately may invite major blackouts, and they should be reported to the system operator (SO) and a control action should be initiated automatically and immediately through global control system [18].

New devices such as Phasor Measurement Units (PMUs) help in Wide Area Measurement and Control. Also, some of the utilities take help of Geographical Information Systems (GIS) for locating the fault and initiating control action. Figure 1 depicts various advanced control devices used for controlling the renewable energy based SG. The brief description of these advanced control devices are described in Section 2.1.

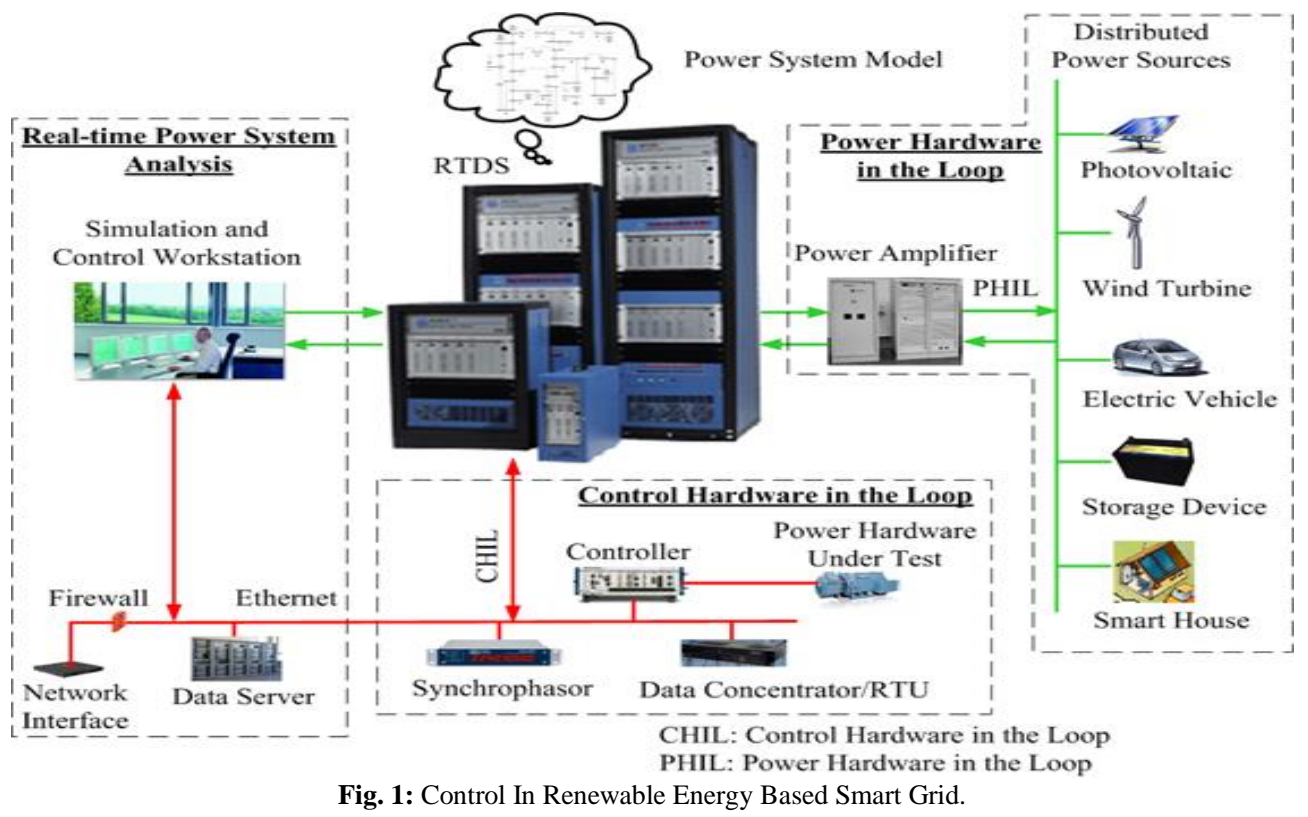

\subsection{Advanced control devices}

\subsubsection{Wide-area measurement systems (WAMS)}

The modern superposition and control tools are required to increase the utilization, for example WAMS are used to allow the networks to operate closer to its capacity while maintaining the system security. The WAMS technology is used to obtain the data and also to extract value from it. By using the WAMS, one can constantly monitor the system and an instant/immediate action can be taken if some failures are detected, and this is the major advantage when compared to the Supervisory control and data acquisition (SCADA) system. These are required, especially to avoid the system-wide black outs. WAMS enables us to observe the power system synchronously in more elaborate time scale. They need data to be sent and captured at very fast rate.

The WAMS consists of phasor measurement unit (PMU) and phasor data concentrator (PDC). PMU uses synchronization signals from the global positioning system (GPS) and provides the phasor voltages and currents measured at substation. PDC is a node in the system, where the phasor data from a number of PMUs or PDCs correlated and fed out as a single stream to other applications. It performs real-time monitoring, quality checks on phasor data, local archiving, alarming and event triggering [19].

\subsubsection{Advanced metering infrastructure (AMI)}

AMI technology is the integration of many technologies which provides an intelligent connection between the consumers and system operators. By using the AMI data, the SOs can improve the customer service by refining the asset management and operating processes of the system. The AMI system has automatic meter reading (AMR) and meter data management (MDM) functions. It provides the link between the generation and storage resources; and utility grid and customer loads. This kind of link is the requirement of a modern grid. The main functions of AMI include remote meter programming, hourly remote meter reading, load control, price signals sent to the customers, customer voltage measurement and outage detection [20].

\subsubsection{Advanced distribution management system (ADMS)}

The ADMS is a unique product which has various fundamental parts such as basic distribution management system (DMS), energy management system (EMS), power control system (PCS), outage management system (OMS), demand response management (DRM) and supervisory control and data acquisition (SCADA). 
The clarity provided by the DMS helps to improve the operator efficiency, increased usage, improved reliability and control room operations. DMS plays a vital role in system integration and outage prevention. The ADMS is formed based on the SG solution concept [21].

\subsubsection{Outage management systems (OMSs)}

The OMS is a computer based software system used by the SOs to reduce outage times and to help system restoration. It uses geographic information system (GIS), AMI and SCADA and the customer information systems (CIS). OMSs are used by the utilities to aid in system handling and repair. OMS includes the computer program to help the decision making process by assessing where the trouble exist, how extensive the outage is, and helps to identify the affected customers, prioritize restoration, calculating restoration time and managing crews and other resources [22].

\subsubsection{Energy management systems (EMSs)}

EMS is required for the industries to use less energy for the same level of activity and also to monitor and control. By using EMS software, one can monitor different areas of various departments which are consuming high energy. EMS gives the clear picture of energy costs, and it helps to optimize and monitor the electrical parameters to achieve cost reduction. The advantages of EMS include easy configuration and maintenance, maximum power demand analysis, and the graphical display of energy consumption By using the EMS, better planning of contingencies is possible The contingency module of EMS helps to handle the emergency online. Improved SCADA system s possible by using the EMS output and hence the improved decision making process is possible in real-time.

\subsubsection{Intelligent electronics devices (IEDs)}

The IEDs are used to increase the availability, power quality in distribution systems. They have several functionalities such as metering, monitoring, protection, serial communication, control functions and logics. The data collected by the IEDs from the customers and the network allows the network reconfiguration locally or by the command from the control center.

\subsection{System security controls}

The security constraints that need to be considered in the renewable energy based SG are presented next:

\subsubsection{Overvoltage control}

The SG should have self-healing and self-restoration qualities. It should monitor at each single corner of the system for abnormalities to self-heal or self-restore. Therefore, it needs to check voltage, current, phase angle, and frequency at each node of the grid. And, also it should compare the obtained quantity with the over voltage, over current quantities at that particular node. If the abnormalities were found, the control action should be initiated immediately and automatically. For the global control, PMU can help to measure voltage and phase angle for wide area. For the local control, CTs and PTs, various meters, transducers connected at each level can help to initiate relay, circuit breaker or any other protective device operation [23].

Moreover, the SGs will be inclusive of stochastic behavior due to the RESs and randomly changing loads. Hence, to carry out the control operation in this environment it requires calculating the dynamic optimal power flow continuously. This can be achieved with the help of next generation optimization tools such as adaptive dynamic programming (ADP), stochastic programming (SP), mixed integer programming (MIP), evolutionary or heuristic programming [24]

\subsubsection{Security measures}

Security measures involve addition of latest devices into the system such as PMUs. PMUs can measure quantities of voltage and phase angle for large area. It can also compare quantities of one area with other and that can help to catch abnormality immediately. It also allows monitoring through the graphs, signals, and charts, etc. Hence, the monitoring is easy and convenient. We can also take help of latest optimization tools to calculate the power flow and optimal power flow (OPF). That will enhance the system with ideal conditions. Therefore, we can easily find abnormal values at each end and it can be corrected before it becomes an issue of major concern. While managing the system with RESs, we should include the advanced storage options such as supercapacitors, storage batteries, hydrogen storage, hydro storage, compressed air energy storage, flywheels, etc. In case of fault, these sources can help to compensate for short coming power needs.

\subsubsection{Contingency analysis}

Contingency analysis is an important part of control system design While designing the SG control system, extra effort should be given to the contingency analysis. Contingency analysis gives the extreme values for line parameters which a system can withstand. Also, through contingency analysis one can rank them to see if one or more abnormalities are going on parallel which should be attended first.

\section{Communication in renewable energy based smart grid}

Communications are the strongest part of SG. In fact, the SG is known by its communication skills. The communication system will be vulnerable to both the physical as well as the cyber-attacks and sufficient layers of security should be provided to handle these vulnerabilities. Moreover, the open source information also direct attacks to the grid and special care and attention should be devoted to these issues before the virtual SG becomes actual SG. Moreover, the grid is vulnerable to natural calamities such as tornadoes, earthquakes, tsunamis, storms and other such situations. These conditions causes major blackout for large areas and it can last for longer duration.

The utility network is Wide Area Network (WAN) with head to toe communication systems involved. The WAN (integrated communications for data transport) has the following components with different functionalities [25]:

- Distributed Energy Resources (DERs): The DERs has distributed generation (DG), RESs and advanced storage technologies.

- Electrical Power Distribution System: It has distribution automation (DA) and microgrids.

- Utility Data Center: It has data storage, management and analysis.

- Utility Control Center: It has Energy Management Systems (EMS), grid monitoring and control applications.

- Power Substation: It has Supervisory Control and Data Acquisition (SCADA) and substation automation networks.

- Customer End: Customers has the ability to access automatic meter reading (AMR), home area networks (HAN), and demand-side management.

The SG involves several real-time devices such as PMUs, smart meters, SCADA, DMS, EMS and many more including real time sensors and power electronics devices. They work altogether to achieve the SG objectives. Real time measurements, monitoring and control are the special features of SG. It involves latest communication and real-time devices that makes the control operation fasters and facilitates the grid with self-healing and self restoration qualities. 


\subsection{Geographical information system (GIS)}

Spatial data systems, including the GIS, have been used successfully by the utility companies, government agencies and many other industries for more than three decades. Systems based on spatial technology have a positive impact on a wide variety of business processes, ranging from marketing to environmental management. Spatial systems bring geography into play within the business processes of a company or organization while enabling data to be visualized more effectively.

SG analytical software such as DMS and outage management systems require high-quality data to provide the right answers. By using the functionality inherent in GIS, the SG technology will start with better-quality data and should, in turn, produce betterquality analytics and operational decisions. In this new SG environment, the state estimation (SE) should include the features such as capable of detecting topology errors, detecting multiple bad data, detecting combinations of three types (topology, parameter, measurement value) of bad data, solving leverage point problem, solving short line problem, solving interacting and confirming bad data, and capable of on-line applications [26].

\subsection{Phasor measurement unit (PMU)}

PMU uses synchronization signals from the global positioning system (GPS) and provides the phasor voltages and currents measured at substation. PMUs can have different data rates such as 10, 30, $60 \mathrm{frames} / \mathrm{sec}$. As PMUs use the GPS system, it is accurate to within 1 microsecond at any location on earth. PMUs provide wide-area situational awareness for SOs, improves the state estimation and accuracy of EMS applications as direct data is more accurate and overcomes the modeling delays. PMUs also have the capability of post-disturbance capability. PMUs provide the synchronized wide-area power measurements. They present measurements as frequency as 30 times in each second. For the SG development at transmission level, PMU deployment plays a vital role for achieving SG goals such as increased system security, efficiency and reliability. PMUs measure synchronously various components such as phase voltages and currents, positive sequence voltages and currents, local frequency and the rate of change of frequency and circuit breaker status, etc [27-28].

\subsection{Remote terminal unit (RTU)}

The SCADA system collects measurements data and the status from the process and it also remotely intervene in the process. RTU is a microprocessors controlled device that interfaces objects in the practical world to a SCADA by transmitting data to the system. The architecture of RTU consists of CPU, volatile and non-volatile memory for processing and storing programs and data RTU collects the site data and sends it to the station via a communications system. Hardware module of RTU includes CPU, analog input and output module, digital input modules, power supply module and communication interfaces [29].

\subsection{Smart meters}

Smart energy meter gives actual power consumption and accurate billing with real time monitoring of electricity uses. It saves time and also cost effective. Measuring the electricity using the smart meter can control the supply remotely and it can cut-off when it is necessary and it is called as the automated meter reading (AMR) AMR uses the one-way communication, i.e., it collects data only from the electrical meter to the utility. Whereas, the new technology called advanced metering infrastructure (AMI) uses the twoway communication and it collects the data from both mater and consumer. Smart meter collects data locally and transfer via a local area network (LAN) to a collection point. The gathered data is used for billing, service monitoring and planning. In future, the smart meter will be a part of IoT (internet of things) interacting multiple aspects of human needs service to satisfy all such needs [30].

\section{Performance assessments for smart grid}

Performance indices must be calculated and finalized for SG. Different researchers, industry experts calculates performance measure differently and hence there is not a unique term exists which can state SG performance. SG performance should be evaluated in all aspects, i.e., safety, maintenance, operation, control, reliability, vulnerability, stability, resiliency, etc. and if possible a unique performance index should be developed that can state performance of the grid with respect to all above parameters. Various performance assessments of SG are presented next [31]:

\subsection{Voltage control}

Voltage control can be obtained through the Var compensators, capacitors, switching of load, installation of new transformer, new line or new substation. Real time Voltage Stability Indices (VSI) can be calculated through the dynamic power flow/OPF or advance devices such as PMU. VSI will give stability limits of the system and we can find out how far is the system from instability [32-33].

\subsection{Frequency control}

Frequency control can be performed through the load frequency control method. Hence, the frequency can be controlled through controlling load, i.e., load shedding, transfer of load, use of available storage options, connection of distributed generation (DG) if possible, etc. Therefore, by controlling the load at the point we can control the frequency.

\subsection{Protection control}

Protection control can be obtained through latest microprocessor relays, circuit breakers and other protecting devices. Operation of these devices can be coordinated with SCADA and EMS for better protection.

\subsection{Stability control}

Voltage regulator is a device that maintains constant voltage. Supply voltage fluctuations have emerged as a serious cause for concern in high performance processor design. Stability control can be achieved through various performance indices, which gives the measure of system stability such voltage stability index, angle stability index, vulnerability index, reliability index, etc. The SG is a virtual concept and only a few working SG in the world. However, before it becomes a reality, a model needs to be developed for it, which can mimic its behavior, issues, challenges and its operation in real time. This model should take into consideration all characteristics of the SG. It will help to improve performance on the SG. This model will be inclusive of all the mathematical relations exist in SG from simple line parameters to state estimation (SE) and PMU.

\section{Cost benefit analysis of control and com- munication in smart grid}

If we consider all the equipments that need to be installed for communication and control operation can be found out the cost benefit analysis (CBA) for the SG, i.e., PMU costs, smart meters costs, relative communication infrastructure, relative protection systems, RTU, etc. if we consider cost for all of this and compare it with the benefits obtained through the installation of these devices. This methodology is based on the use of computer models that simulate the specific characteristics and behaviors of repre- 
sentative parts of SG. Worst case analysis should also be carried out to have a better idea of CBA, i.e., we should consider that after installing everything if grid performance doesn't changes much in such situation benefits obtained will be minimal. Physical benefits can also be converted into societal benefits or utility economic benefits. CBA should be carried out under different situation and with different contingencies applied [34-36].

If we consider all the equipment that needs to be installed for communication and control operation we can find out the CBA for the SG, i.e., PMU costs, smart meters costs, relative communication infrastructure, relative protection systems, RTU, etc., if we consider cost for all of this and compare it with the benefits obtained through installation of these devices. This methodology is based on the use of computer models that simulate the specific characteristics and behaviors of representative parts of SG. Worst case analysis should also be carried out to have a better idea of CBA, i.e., we should consider that after installing everything if grid performance doesn't changes much in such situation benefits obtained will be minimal. Physical benefits can also be converted into societal benefits or utility economic benefits. CBA should be carried out under different situation and with different contingencies applied [37].

\section{Conclusions}

Novel control strategy and communication systems are major part of the smart grid (SG). The bulk power system is facing tremendous challenges in operation and planning as a large number of renewable/distributed energy sources are integrated into the system. With advent of new technology and tools, the grid becomes smart at the same time these tools and technologies adds relative complexity to power system behavior as well. This paper presents the challenges and issues related to the control and communication for SG. The next-generation control architecture for future power grid with massive renewable energy sources (RESs) is presented in this paper. It provides the state-of-the-art RESs into the system and the recent control and communication technologies in the renewable energy sources based SG. The cost benefit analysis (CBA) is carried out by different researchers motivates the investment done for control and communication technologies in SG.

\section{Acknowledgment}

This research work is based on the support of "Woosong University's Academic Research Funding - 2018".

\section{References}

[1] K.S. Reddy, M. Kumar, T.K. Mallick, H. Sharon, S. Lokeswaran, A review of Integration, Control, Communication and Metering (ICCM) of renewable energy based smart grid, Renewable and Sustainable Energy Reviews, vol. 38, (2014), pp. 180-192. https://doi.org/10.1016/j.rser.2014.05.049.

[2] M.L. Tuballa, M.L. Abundo, A review of the development of Smart Grid technologies, Renewable and Sustainable Energy Reviews, vol 59, (2016), pp. 710-725. https://doi.org/10.1016/j.rser.2016.01.011.

[3] M.S. Hossain, N.A. Madlool, N.A. Rahim, J. Selvaraj, A.K. Pandey, A.F. Khan, Role of smart grid in renewable energy: An overview, Renewable and Sustainable Energy Reviews, vol. 60, (2016), pp. 1168-1184. https://doi.org/10.1016/j.rser.2015.09.098.

[4] E.F. Camacho, T. Samad, M. Garcia-Sanz, I. Hiskens, Control for Renewable Energy and Smart Grids, 2011. Available. [Online]: https://web.eecs.umich.edu/ hiskens/publications/IoCT-Part106RESG.pdf.

[5] M.A. Mohamed, A.M. Eltamaly, H.M. Farh, A.I. Alolah, Energy management and renewable energy integration in smart grid system IEEE International Conference on Smart Energy Grid Engineering (SEGE), Oshawa, ON, (2015), pp. 1-6. https://doi.org/10.1109/SEGE.2015.7324621.

[6] Smart Grids and Renewables - A Guide for Effective Deployment, Working Paper, Nov. 2013. Available. [Online] https://www.irena.org/documentdownloads/publications/smart_grid s.pdf.

[7] N. Uribe-Pérez, I. Angulo, D. de la Vega, T. Arzuaga, I. Fernández, A. Arrinda, Smart Grid Applications for a Practical Implementation of IP over Narrowband Power, Line Communications, Energies, vol. 10, pp. 1-16, 2017. https://doi.org/10.3390/en10111782.

[8] Y. Li, F. Nejabatkhah, Overview of control, integration and energy management of microgrids, Journal of Modern Power Systems and Clean Energy, vol. 2, no. 3, (2014), pp. 212-222. https://doi.org/10.1007/s40565-014-0063-1

[9] M.A. Islam, M. Hasanuzzaman, N.A. Rahim, A. Nahar, M. Hosenuzzaman, Global Renewable Energy-Based Electricity Generation and Smart Grid System for Energy Security, The Scientific World Journal, vol. 2014, (2014), pp. 1-13. https://doi.org/10.1155/2014/197136.

[10] S.M. Muyeen, S. Rahman, Communication, Control and Security Challenges for the Smart Grid, IET, 2017.

[11] S. Mathavi, D. Vanitha, S. Jeyanthi, P.S. Kumaran, The Smart Home: Renewable Energy Management System for Smart Grid Based On ISM Band Communications, International Journal of Scientific \& Engineering Research, vol. 3, no. 3, (2012), pp. 1-8.

[12] M.E. El-hawary, The Smart Grid - State-of-the-art and Future Trends, Electric Power Components and Systems, vol. 42, no. 3-4 pp. $239-250$ https://doi.org/10.1080/15325008.2013.868558

[13] B.S. Hartono, S.P. Mursid, S. Prajogo, Review: Home energy management system in a Smart Grid scheme to improve reliability of power systems, IOP Conf. Series: Earth and Environmental Science, vol. 105, (2018), pp. 1-10. https://doi.org/10.1088/17551315/105/1/012081.

[14] B. Speer, M. Miller, W. Shaffer, L. Gueran, A. Reuter, B. Jang, K. Widegren, The Role of Smart Grid in Integrating Renewable Energy, Technical Report, National Renewable Energy Laboratory, May 2015.

[15] Y. Kabalci, A survey on smart metering and smart grid communication, Renewable and Sustainable Energy Reviews, vol. 57, (2016), pp. 302-318. https://doi.org/10.1016/j.rser.2015.12.114.

[16] A. Naamane, N.K. Msirdi, Towards a Smart Grid Communication, Energy Procedia, vol. 83, (2015), pp. 428-433. https://doi.org/10.1016/j.egypro.2015.12.162.

[17] Y.W. Law, H.R. Pota, J. Jin, Z. Man, M. Palaniswami, Control and Communication Techniques for the Smart Grid: An Energy Efficiency Perspective, IFAC Proceedings Volumes, vol. 47, no. 3, (2014), pp. 987-998.

[18] S. Alam, M.F. Sohail, S.A. Ghauri, I.M. Qureshi, N. Aqdas, Cognitive radio based Smart Grid Communication Network, Renewable and Sustainable Energy Reviews, vol. 72, (2017), pp. 535-548. https://doi.org/10.1016/j.rser.2017.01.086.

[19] B. Zhang, J. Baillieul, Communication and Control Protocols for Load Networks in the Smart Grid, IFAC Proceedings Volumes, vol. 47, no. 3, (2014), pp. 11250-11256.

[20] Available. [Online]: https://www.smartgrid.gov/files/NIST_SG_Interop_Report_Postco mmentperiod_version_200808.pdf

[21] Available. [Online]: https://www.schneider-electricdms.com/media/SEDMS\%20SW\%20Short\%20Overview.pdf

[22] J. Gao, Y. Xiao, J. Liu, W. Liang, C.L.P. Chen, A survey of communication/networking in Smart Grids, Future Generation Computer Systems, vol. 28, no. 2, (2012), pp. 391-404. https://doi.org/10.1016/j.future.2011.04.014.

[23] E. Ancillotti, R. Bruno, M. Conti, The role of communication systems in smart grids: Architectures, technical solutions and research challenges, Computer Communications, vol. 36, no. 17-18, (2013), pp. 1665-1697. https://doi.org/10.1016/j.comcom.2013.09.004.

[24] R. Miceli, Energy Management and Smart Grids, Energies, vol. 6, (2013), pp. 2262-2290. https://doi.org/10.3390/en6042262.

[25] A. Chakrabortty, M.D. Ilic, Control and Optimization Methods for Electric Smart Grids, Power Electronics and Power Systems, Springer, 2012

[26] Z. Fan, et al., Smart Grid Communications: Overview of Research Challenges, Solutions, and Standardization Activities, IEEE Communications Surveys \& Tutorials, vol. 15, no. 1, pp. 21-38, First Quarter, 2013.

[27] V. Padmini, S. Omran, K. Chatterjee, S.A. Khaparde, Cost benefit analysis of smart grid: A case study from India, North American Power Symposium (NAPS), Morgantown, WV, (2017), pp. 1-6. https://doi.org/10.1109/NAPS.2017.8107212.

[28] O.P. Mahela, A.G. Shaik, Comprehensive Overview of Grid Interfaced Wind Energy Generation Systems, Renewable and Sustaina- 
ble Energy Reviews, vol. 67, (2016), pp. 260-281 https://doi.org/10.1016/j.rser.2015.12.048.

[29] F.E. Idachaba, A. Ogunrinde, Review of Remote Terminal Unit (RTU) and Gateways for Digital Oilfield delpoyments, International Journal of Advanced Computer Science and Applications, vol. 3, no. 8 , (2012), pp. 157-160.

[30] N. Karali, C. Marnay, T.S. Yan, G. He, K. Clampitt, R. Yinger, J. Mauzey, H. Zhu, Towards uniform benefit-cost analysis for smart grid projects: an example using the Smart Grid Computational Tool, Technical Report, Dec. 2015. Available. [Online]: https://eta.lbl.gov/sites/all/files/publications/lbnl-1003908_isgdtowardsuniformcba.pdf

[31] Z. ul Abideen, S. ul Hassan, Enabling smart grids through advanced communication and control, IEEE International Conference on Smart Grid and Smart Cities (ICSGSC), Singapore, (2017), pp. 189-194.

[32] S.Surender Reddy, James A. Momoh, "Feasibility of Stochastic Voltage/VAr Optimization Considering Renewable Energy Resources for Smart Grid", International Journal of Emerging Electric Power Systems, vol. 17, no. 3, pp. 287-300, Jun. 2016.

[33] J.A. Momoh, S. Surender Reddy, Y. Baxi, "Stochastic Voltage/Var Control with Load Variation", IEEE PES General Meeting, Jul. 2014, Washington, DC, National Harbor, MD, USA, pp. 1-5.

[34] Z.X. Zou, G. Buticchi, M. Liserre, Control and communication in the Smart Transformer-fed grid, IEEE 21st International Conference on Emerging Technologies and Factory Automation (ETFA), Berlin, (2016), pp. 1-9.

[35] S.R. Salkuti, Overview and tools of asset management in renewable energy based smart grid, International Journal of Engineering \& Technology, vol. 7, no. 2.27, (2018), pp. 22-26.

[36] S.R. Salkuti, Large scale electricity storage technology options for smart grid, International Journal of Engineering \& Technology, vol. 7, no. 2, (2018), pp. 635-639.

[37] C.I. Ciontea, R. Pedersen, T. Le Fevre Kristensen, C. Eg Sloth, R.L. Olsen, F. Iov, Smart grid control and communication: The SmartC2net Real-Time HIL approach, IEEE Eindhoven PowerTech, Eindhoven, (2015), pp. 1-6. 